\title{
Caracterización socioeconómica y demográfica de niños y adolescentes que estudian y trabajan simultáneamente fuera de casa*
}

\author{
CARACTERIZAÇÃO SOCIOECONÔMICA E DEMOGRÁFICA DE CRIANÇAS E \\ ADOLESCENTES QUE SIMULTANEAMENTE ESTUDAM E TRABALHAM FORA DE CASA
}

\author{
THE SOCIOECONOMIC AND DEMOGRAPHIC CHARACTERIZATION OF CHILDREN AND \\ ADOLESCENTS WHO STUDY AND WORK OUTSIDE THEIR HOME
}

\section{Renata Cristina da Penha Silveira ${ }^{1}$, Luiz Cláudio Ribeiro², lara Aparecida de Oliveira Secco ${ }^{3}$, Maria Lúcia do Carmo Cruz Robazzi ${ }^{4}$}

\section{RESUMEN}

Identificar las características socioeconómicas y demográficas de niños y adolescentes que estudiaban y trabajaban simultáneamente fuera de casa. Estudio no-experimental, correlacional, de delineación transversal. Se aplicó cuestionario a alumnos de enseñanza primaria matriculados en escuelas públicas de Ribeirão Preto-SP (Brasil). Dos escuelas fueron sorteadas. Se utilizó Statistical Package of Social Sciences, versión 14,0 para análisis de datos. De los 133 estudiantes que respondieron el cuestionario, $36(27,7 \%)$ refirieron trabajar fuera de casa, 20,6\% tenía 11 y 13 años, $66,7 \%$ eran de sexo masculino $(p=0,000)$ y comenzaron a trabajar precozmente para sumar a la renta familiar $(p=0,003)$. El salario recibido se aplicaba a la mejora de la renta familiar, constatándose que si dicha renta aumentaba, la presencia del joven en su trabajo disminuía. Se evidenció que muchos factores contribuyen en la inserción precoz de estos sujetos en el mercado laboral, tales como el tamaño, la estructura familiar y la pobreza.

\section{DESCRIPTORES}

Trabajo de menores

Pobreza

Educación primaria y secundaria

Salud laboral

\section{RESUMO}

Identificar as características socioeconômicas e demográficas das crianças e adolescentes que simultaneamente estudavam e trabalhavam fora de casa. Estudo não-experimental, correlacional de delineamento transversal, baseado em questionários aplicados aos alunos do ensino fundamental, matriculados em escolas públicas de Ribeirão Preto (Brasil). Duas escolas foram sorteadas. Para a análise dos dados foi utilizado o Statistical Package of Social Sciences, versão 14.0. Dos 133 estudantes que responderam o questionário, $36(27,7 \%)$ informaram trabalhar fora de casa, 20,6\% tinham entre 11 e 13 anos, 66,7\% eram do sexo masculino $(p=0,000)$ e começaram a trabalhar precocemente para compor a renda familiar $(p=0,003)$. O salário recebido auxiliava na composição da renda familiar, constatando-se que se à medida que a renda familiar aumentava, a presença do jovem no trabalho diminuía. Foram encontradas evidências de que muitos fatores contribuem para a inserção precoce destes sujeitos no mercado de trabalho, como o tamanho, a estrutura da família e a pobreza.

\section{DESCRITORES}

Trabalho de menores

Pobreza

Ensino fundamental e médio

Saúde do trabalhador

\begin{abstract}
The objective of this study was to identify the socioeconomic and demographic characteristics of children and adolescents who study and work outside their home. This non-experimental, correlational, cross-sectional study was performed using questionnaires applied to primary education students, enrolled in public schools in Ribeirão Preto (Brazil). Two schools were selected through a draw. Data analysis was performed using Statistical Package for Social Sciences, version 14.0. Of the 133 students who answered the questionnaire, 36 (27.7\%) reported working outside their home, $20.6 \%$ were between 11 and 13 years of age, and $66.7 \%$ were male $(p=0.000)$ and had started working early to help with the family income $(p=0.003)$. The salary they received helped comprise the family income, and it was found that as the family income increased, the need for the youngsters to work was reduced. It was found that many factors contribute to these subjects' early start at work, including family size, structure and poverty.
\end{abstract}

\author{
DESCRIPTORS \\ Child labor \\ Poverty \\ Education, primary and secundary \\ Occupational health
}

\footnotetext{
* Extraído de la tesis "Repercusiones del trabajo en el aprovechamiento escolar de niños y adolescentes: retrato de una realidad", Programa de Postgrado en Enfermería de la Escuela de Enfermería de Ribeirão Preto de la Universidad de São Paulo, 2009. ${ }^{1}$ Enfermera. Profesor Adjunta de la Universidad Federal de São João Del Rei del Campus Centro-Oeste Dona Lindu. Divinópolis, MG, Brasil. renatacps@hotmail.com ${ }^{2}$ Ingeniero. Profesor Adjunto del Instituto de Ciencias Exactas de la Universidad Federal de Juiz de Fora. Juiz de Fora, MG, Brasil. luizclaudio@ice.ufjf.br ${ }^{3}$ Enfermera. Responsable Técnica en el Hospital Universitario de Londrina de la Universidad Estatal de Londrina. Londrina, PR, Brasil. iarasecco@sercomtel.com.br ${ }^{4}$ Enfermera. Profesor de la Escuela de Enfermería de Ribeirão Preto de la Universidad de São Paulo. Ribeirão Preto, SP, Brasil. avrmlccr@eerp.usp.br
} 


\section{INTRODUCCIÓN}

El trabajo del niño y del adolescente hacen años que está presente en el día a día de las personas, esta mano de obra parece haber participado activamente en el proceso de desarrollo de las antiguas civilizaciones. Según el articulo 2ㅇ del Estatuto del Niño y Adolescente (Ley $\mathrm{n}$ 8.069, de 13/07/90) en el título I, Disposiciones Preliminares, se entiende por niño: $A$ la persona hasta 12 años de edad incompletos y adolescente entre 12 y 18 años de $e d a d^{(1)}$. Para la Organización Mundial de la Salud (OMS) citado por la Organización Panamericana de la Salud; en 1990, son considerados adolescentes (...) todos aquellos comprendidos en el grupo de edad entre los diez y veinte $a_{n} o^{(2)}$. A partir del año 2000, según la ratificación de la Organización Internacional del Trabajo (OIT), firmada por el Brasil, se considera como menor de edad a todo trabajador con menos de 18 años completos y considerándose como edad mínima para el trabajo de 16 años, esto se mantiene desde la reunión de la Cámara de Diputados realizada el año $2009^{(3)}$.

No obstante estar prohibido prácticamente en todos los países, en recientes investigaciones de la $\mathrm{OIT}^{(4)}$ se detectó que hay cerca de 165 millones de niños de cinco a 14 años de edad en el mundo, comprometidos con alguna forma de trabajo. El trabajo infantil peligroso, o sea, trabajos vinculados a la dirección de vehículos motorizados, mantenimiento o limpieza de máquinas y equipos cuando estén en movimiento, así como el manejo de tractores agrícolas, máquinas de cortar, (...) trabajos de construcción civil o construcción pesada, afilar herramientas, (...) trabajos en la operación industrial de reciclado de papel, plástico o metal, entre otros, estos afectan a cerca de 115 millones de niños (60\% de niños) lo que representa más de la mitad de todos los trabajadores infantiles del mundo, estimados en 215 millones. De tal forma el número de niños entre 15 y 17 años en este tipo de actividades tuvo un aumento real de $20 \%$ de 2004 para 2008, pasando de 52 millones para 62 millones. La OIT hace un llamado para que nuevos esfuerzos para asegurar que todos los niños tengan acceso a la educación por lo menos hasta la edad mínima para ser empleados (...) y son necesarias medidas urgentes para combatir el trabajo peligroso en niños que hayan llegado a la edad mínima, y al mismo tiempo pueden estar en riesgos de accidentes de trabajo y enfermedades ocupacionales. Así la OIT pide entrenamiento para estos jóvenes trabajadores de manera que estén conscientes de los riesgos, derechos y posibilidades en el local de de trabajo.

La misma organización informa, que (...) las niñas constituyen el mayor número de menores en trabajos domésticos en hogares de terceros. Existen denuncias regulares respecto a avisos de niñas en trabajos domésticos. En sus propios hogares las niñas desempeñan labores domésticas con más frecuencia que los niños. Esto sumado a la actividad económica fuera del hogar, representa una doble carga que aumenta el riesgo de que las niñas abandonen la escuela(5).

Mundialmente el número de niños inmersos en actividades económicas es muy grande; en África es casi 50 millones, lo que representa un $26 \%$ del número total de niños; la mayor cantidad se presentan en la región Asia-Pacífico donde 122 millones, con edades comprendidas entre cinco y 14 años ya trabajan. En América Latina y el Caribe se tienen progresos hay una disminución del 5\% de niños entre cinco y 14 años envueltos en actividades laborales fuera de casa ${ }^{(6)}$.

El Brasil esta empeñado en la erradicación del trabajo infantil, en la década de los 90's del siglo pasado, fue aprobado el Estatuto da Criança e do Adolescente (ECA) ${ }^{(1)}$. Este documento describe la prohibición de dar cualquier trabajo a menores de 14 años de edad, salvo en la condición de aprendiz ${ }^{(1)}$. Actualmente la legislación nacional prohíbe el trabajo nocturno, peligroso o insalubre a los menores de 18 años y cualquier actividad otra laboral a los que tengan edad inferior a los 16 años salvo en la condición de aprendiz ${ }^{(7)}$. El Estatuto prohíbe también el trabajo en minas, estibaje, o cualquier trabajo subterráneo para aquellos por debajo de los 21 años.

A pesar de estas disposiciones en el Brasil según la Investigación Nacional por muestra de Domicilios (PNAD) se estima que en el año 2009, 2,0 millones de niños de 5 a 15 años de edad ocupadas en el mercado de trabajo, de los cuales cerca del $44 \%$ estaban concentrados en la Región Nordeste y $24 \%$, en la Región Sudeste. Considerando apenas su forma más grave, fueron 122679 niños de 5 a 9 años encontrados en situación de trabajo infantil, casi la mitad de ellas en la región Nordeste ${ }^{(8)}$.

Para la OIT, la exposición de riesgos puede tener un impacto particularmente grave en niños, cuyos cuerpos y mentes todavía no están desarrollados en el final de la adolescencia. El informe examina en detalles seis sectores económicos: agropecuario, pesca, trabajo doméstico, minas, trabajo infantil urbano y en servicios. El estudio observa el problema de los niños en trabajos peligrosos no se limita a los países en desarrollo. Existen evidencias que en los Estados Unidos y en Europa también hay un número elevado de vulnerabilidades de los jóvenes para Accidentes de Trabajo (AT); un número substancial de niños que pasan largas horas de trabajo, lo que aumenta significativamente el riesgo de accidentes; mayor número de niños en trabajos peligrosos está en los países asiático y del Pacífico, (...) el trabajo peligro es más comúnmen- 
te encontrado en al agricultura, incluyendo la silvicultura, pesca, pecuaria, e acuicultura, además de agricultura de subsistencia y la agricultura comercial ${ }^{(4)}$.

El trabajo del niño y del adolescente esta directamente vinculado a la pobreza del brasileño. En general, una familia con bajos ingresos no puede disponer de los medios suficientes para garantizar la educación de los niños. Además de eso, la familia depende de la contribución financiera que el niño trabajador pueda traer para completar los ingresos familiares y se considera tal contribución más importante que el acceso a la educación. Cuando una familia tiene que escoger y enviar los hijos a la escuela, prefiere que sean los niños antes que las niñas ${ }^{(4)}$.

La mayoría de los niños y adolescentes cuya mano de obra es explotada, trabaja 25 horas semanales lo cual representa el $44,8 \%$ y casi el $30 \%(28,6 \%)$, ocupa hasta 40 horas semanales en actividades laborales. Por este motivo casi $25 \%$ de los niños y adolescentes que trabajan, dejan la escuela ${ }^{(9)}$.

Como resultado y consecuencia de la pobreza, el trabajo realizado por los niños y adolescentes perpetúa situaciones desfavorables y de exclusión social, al impedir la escolarización y/o perjudicar el aprovechamiento escolar de los niños, se limita a la obtención de conocimientos y actitudes necesarios para que, como adultos, puedan contribuir al crecimiento económico prosperidad, lo cual retara el desarrollo nacional ${ }^{(10)}$. Al observar la posición de la sociedad brasileña en relación al trabajo de los niños, se percibe que existe un apoyo de las familias, habiendo consecuentemente una naturalización del trabajo infantil, considerándolo como una solución a las carencias del sistema educativo, la pobreza y la violencia en las calles. Por lo expuesto anteriormente, el objetivo de este estudio es identificar las características socioeconómicas y demográficas de los niños y adolescentes que simultáneamente estudian y trabajan fuera de sus casas

\section{MÉTODO}

Estudio no experimental y transversal, que incluyó alumnos de enseñanza primaria (a partir de la quinta serie) ${ }^{(7)}$. Fue realizado en la ciudad de Ribeirao Preto, situada en la región Noroeste del Estado de Sao Paulo, Brasil, a 313 KM de la capital paulista. De acuerdo con los datos de la Cámara Municipal de Ribeirao Preto y el informe del Instituto Brasileiro de Geografía y Estadística (IBGE), en el censo de la población efectuado en el año 2006, presentaba 42,840 alumnos inscritos desde la educación infantil hasta la educación profesional. En la enseñanza primaria (de 5 a a $8^{\text {a }}$ serie) el número de alumnos fue de $10,627^{(11)}$. La población estudiada fue compuesta por alumnos inscritos en escuelas de la red municipal de la ciudad de Ribeirao Preto (SP) en el año de 2006. La muestra fue seleccionada aleatoriamente, por conglomerados en un estadio, participando solamente los alumnos matriculados en el nivel prima- rio de 5a a 8 ${ }^{\mathrm{a}}$ serie del municipio. Fue realizado un sorteo a través del programa Statical Package of Social Sciencies (SPSS). Las escuelas seleccionadas pertenecían a la zona norte de la ciudad de Ribeirao Preto y fueron denominadas: Escuela $A(E A)$ y Escuela $B(E B)$, en relación a los aspectos éticos se consideró las directrices y Normas Reglamentarias de Investigación en seres humanos, emanados de la Resolución 196 del año 1996 del Consejo Nacional de Salud Brasileño(12). El proyecto fue enviado a un Comité de Ética e Investigación de la Universidad de Sao Paulo y fue aprobado (oficio 166/2006). Fue concedida también la autorización de la Secretaria de Educación responsable, para que el estudio fuese realizado en las instituciones seleccionadas, se tuvo también el consentimiento por parte del Juez de la Infancia y de la Juventud para la realización de la investigación. Posteriormente la investigadora un día antes de la aplicación del cuestionario, explicó en la sala de clase, los objetivos de la investigación a los alumnos, posteriormente, el consentimiento libre e informado fue proporcionado a los padres o responsables legales de los alumnos y solamente después de la firma de los mismos se permitió la participación del niño/adolescente en la investigación, así como la firma del estudiante así el cuestionario fue aplicado. Como criterios de selección de los encuestados se tomó en cuenta los siguientes factores: tener edad inferior a 18 años, estar inscritos en las escuelas seleccionadas y estar presente en la sala de clases o en la escuela en el momento de la aplicación del cuestionario. El cuestionario contenía preguntas abiertas y cerradas que abordaron las características socioeconómicas y demográficas de los niños/ adolescentes, de su familia y del trabajo realizado por los mismos fuera de sus casas.

Para el análisis de las variables se elaboró un diccionario (codebook) así como un banco de datos, empleándose el programa Excel para Windows 2003, con el objetivo de evitar errores de digitación se realizó doble digitación, validación y confirmación de los datos. Posteriormente todos los datos fueron pasados al programa SPSS versión 14.0 para realizar análisis estadísticas descriptivas (media, mediana) y de variabilidad (desviación estándar) además de análisis univariados y bivariados. Fue previamente determinado por el nivel de significación del 5\%. Para la comparación de las medias de las edades, del número de hermanos, entre los grupos que trabajaba fuera casa y de los que no trabajaban fuera de casa, dado que en ambos, el número de sujetos fue mayor que 30 , se utilizó la prueba estadística paramétrica $t$ de Student.

Para la variable: sujetos que trabajan fuera de casa, el número fue menor que 30 por tanto se utilizó la prueba no paramétrica de Kolmogorov-Smirnov, a fin para verificar la normalidad de la distribución de las respuestas. En el caso de las variables categóricas la prueba no paramétrica Chi Cuadrado fue utilizado para probar la homogeneidad de las proporciones de las respuestas entre los grupos que trabajaban en casa y fuera de ella. Cuando las frecuencias fueron menores o iguales a cinco se utilizó la
Caracterización socioeconómica y demográfica de niños y adolescentes que estudian y trabajan simultáneamente fuera de casa Silveira RCP, Ribeiro LC, Secco IAO, Robazzi MLCC 
prueba exacta de Fischer. Se adopto un nivel de significancia alfa =0,05 para la aplicación de las pruebas estadísticas. Cuando el valor de la significancia calculada ( $p$ ) fue menor a 0,05 la diferencia entre las asociaciones fueron consideradas estadísticamente significativas.

\section{RESULTADOS}

\section{Datos Generales}

De los 133 estudiantes que respondieron el cuestionario y que cumplían los criterios de inclusión, la mayoría $(63,2 \%)$ eran del sexo femenino, tenían como edad minina 10 años y máximo 16 años; la mayoría (34 niños) tenían 14 años (25,6\%), seguido por niños con 11 años $(23,3 \%)$ y 13 años de edad (23,3\%), mulatos $(45,9 \%)$ padres con empleo (84\%), con hermanos $(95,5 \%)$, residentes en casa propia (74,2\%), 95,5\% informaron ayudar a los padres en casa, hasta aquellos que trabajaban fuera de casa.

\section{Características de los estudiantes trabajadores}

De los 36 (27,7\%) niños estudiantes que informaron trabajar fuera de casa, la edad mínima informada iniciada en el trabajo, varió desde los seis años de edad (dos casos) hasta 15 años (tres casos). Entre tanto el mayor número de niños (20,6\%) inició las actividades laborales a los 11 y 13 años de edad (20,6\%), la edad más frecuente fue a los 12 años (17,6\%), seguida por $14,7 \%$ que iniciaron el trabajo con 14 años de edad, 8,4\% que iniciaron el trabajo con 15 años de edad, entre otros. En relación al sexo, $66,7 \%$ eran de sexo masculino, y al realizar la prueba de Chi Cuadrado se constató que en relación al sexo, hay una tendencia para que el porcentaje de adolescentes trabajadores sea mayor al de las adolescentes $p=0,000$.

En relación al color de la piel de los 36 niños trabajadores $50 \%$ eran mulatos, al realizar la prueba de Chi Cuadrado se comprobó que el color de la piel $p>0,05(p=0.803)$ no mostró diferencia estadísticamente significativa.

Al realizar la estadística descriptiva de las variables cuantitativas y la variable trabajo fuera de la casa fue posible resaltar lo que se presenta en la Tabla 1.

Tabla 1 - Comparación de las variables entre los grupos de niños/adolescentes que trabajan fuera de casa y el grupo de niños adolescentes que no trabajan fuera de casa - Ribeirão Preto, SP, Brasil - 2008

\begin{tabular}{|c|c|c|c|c|c|c|c|c|}
\hline \multirow{2}{*}{ Variables } & \multicolumn{3}{|c|}{ Trabaja fuera } & \multicolumn{3}{|c|}{ No trabaja fuera } & \multirow[b]{2}{*}{$\mathbf{t}$} & \multirow[b]{2}{*}{$\mathbf{P}$} \\
\hline & $\mathbf{n}$ & Media & D E & $\mathbf{n}$ & Media & D E & & \\
\hline Edad (en años) & 36 & 13,3 & 1,5 & 94 & 12,6 & 1,3 & 2,779 & 0,003 \\
\hline Número de hermanos & 33 & 3,3 & 1,2 & 91 & 3,2 & 1,9 & 0,429 & 0,669 \\
\hline Ingreso en U\$ & 22 & 338,4 & 498,2 & 60 & 424,7 & 248,4 & $-1,02$ & 0,309 \\
\hline $\begin{array}{l}\text { Horas que ayudan a los padres } \\
\text { en casa/día }\end{array}$ & 30 & 3,4 & 2,9 & 85 & 3,6 & 2,1 & $-0,317$ & 0,752 \\
\hline $\begin{array}{l}\text { Edad de inicio de ayuda en el tra- } \\
\text { bajo en casa }\end{array}$ & 26 & 8,7 & 2,5 & 85 & 9,2 & 1,8 & $-0,808$ & 0,424 \\
\hline $\begin{array}{l}\text { Edad de inicio en el trabajo fuera } \\
\text { de casa }\end{array}$ & 34 & 11,9 & 2,2 & - & - & - & - & - \\
\hline Salario (en reales) & 21 & 98,6 & 125,4 & 21 & 18,4 & 19,1 & - & - \\
\hline
\end{tabular}

Se observa en la Tabla 1, que los niños/adolescentes que trabajan fuera de casa, en general, el promedio de edad fue de 13,3 años (mediana hasta de 14 años), la media de edad de los que no trabajan fuera de casa fue de 12,6 años (mediana de hasta 13 años) comprobándose que hubo variación estadística para la edad de los sujetos que trabajaban fuera de casa, que eran mas grandes. Al analizar la edad de inicio en el trabajo se constato que el promedio de edad fue de 11,9 años. Los niños con edades entre 14 y 15 años $(23,1 \%)$, trabajaban fueran de casa una media de siete horas en restaurantes, casas de familiares, fábricas, talleres mecánicos, como ayudante de albañil/ pintor entre otros. Los motivos presentados para iniciar el trabajo fuera de casa fueron: ayudar a los padres para aumentar el ingreso familiar (12 casos, 33\%) y ganar dinero (cuatro casos 11,1\%), entre otros. Además de trabajar fuera de casa, $88,9 \%$ ayudaban a los padres en casa después del trabajo, en actividades domésticas como lavar loza, planchar, cuidar de los hermanos más pequeños, entre otras. En cuanto a las horas que los niños ayudaban a los padres en casa, se comprobó que para los grupos que trabajaban fuera de casa, la media de horas de trabajo fue de 3,4hs/día y para los que no trabajan fuera de casa la media fue de 3,6 horas/día, mostrando que ambos grupos presentaban aproximadamente 28 horas de trabajo semanales dentro de casa, estudios consideran que el trabajo para el niño/ adolescente por una hora o más por semana impide que esos niños dediquen tiempo para jugar y realizar actividades infantiles, influenciado su desarrollo dinámico de acuerdo con su edad.

En cuanto al ingreso familiar de los padres de los estudiantes que trabajan fuera de casa, la medía fue de U\$ 338,4 [2,06 salarios mínimos] siendo que para la mitad de esos sujetos, los ingresos fueron hasta U\$ 206,57 [1,25 salarios mínimos], considerando que el valor del dólar era de $\mathrm{R} \$ 2.13$ 


\section{DISCUSIÓN}

Para la OIT en el año 2003, hay un número mayor de niños y adolescentes del sexo masculino que del femenino trabajando, siendo la proporción de tres niños por dos niñas, en promedio. En el Brasil, el número de niñas trabajadoras con frecuencia, es subestimado por los datos estadísticos que generalmente no consideran como trabajo la actividad económica no remunerada desarrollada por las niñas dentro y fuera del hogar familiar ${ }^{(13)}$.

En esta investigación, de los 36 estudiantes que informaron trabajar fuera de casa 33,3\% eran niñas, el porcentaje de niños en el trabajo fue prácticamente el doble (66,7\%).

Corroborando ${ }^{(14)}$, la proporción de niños trabajadores es mayor que el de niñas, excepto en el empleo doméstico, en el que la mayoría de los trabajadores son mujeres. Además, el porcentaje de trabajo infantil en las áreas rurales es más elevado que en las áreas urbanas del Brasil.

El mayor número de sujetos $(20,6 \%)$ inició las actividades laborales entre los 11 y 13 años de edad; la edad más frecuente fue de 12 años (17,6\%) seguida por $14,7 \%$ que se iniciaron en el trabajo con a la edad de 14 años.

Datos semejantes fueron encontrados en la investigación realizada en la cuidad de Pelotas, Río Grande do Sul, Brasil, donde fueron entrevistados 4, 924 individuos entre seis y 17 años de edad, de los 476 niños trabajadores, $70 \%$ tenían entre 14 y 17 años de edad, $25 \%$ entre 10 y 13 años, 5,3\% entre seis y nueve años de edad y $88 \%$ se encontraban trabajando en el mercado informal. Tal hecho muestra que un gran número de niños/adolescentes inicia sus actividades laborales antes de la edad recomendada por las Legislaciones Nacionales ${ }^{(15)}$.

Tal situación se evidencia en el estudio ${ }^{(16)}$ realizado en la ciudad de Río de Janeiro, en un ambulatorio del Programa de Salud del Trabajador Adolescente, demostrando que entre los años 1997 y 2002 fueron atendidos 275 adolescentes, con edad promedio de 16 años, siendo 93,5\% entre 14 años, y 6,5\% entre 18 a 19 años, el promedio de edad para el inicio de las actividad laborales fue 14 años.

Tales datos muestran que la mayoría de los niños y adolescentes trabajan informalmente e inician sus actividades laborales tempranamente.

En cuanto al color de la piel, el presente estudio mostró que la mayoría $(45,9 \%)$ de los sujetos son mulatos (mestizaje entre la raza blanca y la negra) está realidad se encontró en la gran mayoría de los jóvenes que necesitan trabajar en el Brasil.

El género y el color son importantes para determinar las diferentes posibilidades del individuo a tener acceso al empleo y buenas condiciones de trabajo además de remuneraciones, beneficios y posibilidades de protección social. De este modo, tales factores condicionan la forma por la cual los individuos y las familias viven en situación de pobreza y no consiguen superarla(6).

Confirmando, en una investigación realizada utilizando datos del Sistema de Avaliação da Educação Básica (SAEB) de 1999, mostró las diferencias raciales en el Brasil, percibiéndose que los mulatos presentaban un desempeño inferior al de los alumnos blancos en 2,5 puntos. Esa diferencia aumentaba cuando se trataba de negros. Para los autores, la comprobación de las desigualdades es un resultado preocupante que debe ser tomado en cuenta por las autoridades gubernamentales ${ }^{(17)}$.

Siendo así, la probabilidad de los niños blancos para trabajar es menor si se compara con el color de los negros o mulatos, por lo tanto, en la presente investigación, el efecto de las razas no se mostró estadísticamente significativa en la inserción temprana de los niños blancos o negros/ mulatos en el mercado de trabajo. Este hecho muestra la concordancia del estudio con los informes de la literatura, sobre el color de los niños/adolescentes trabajadores ${ }^{(18)}$.

En esta investigación la mayoría de padres de los niños encuestados (84\%) estaba empleado, lo que significa que el joven trabajador no es el único proveedor de recursos financieros en la familia.

Varios factores ${ }^{(14)}$, contribuyen en la inserción de los niños en el mercado de trabajo, como por ejemplo, la pobreza, la escolaridad de los padres, el tamaño y la estructura de la familia, el sexo del jefe, la edad en la que los padres comenzaron a trabajar y el lugar de residencia que explica la dedicación del tiempo del niño para el trabajo.

En el año 2002 el Brasil presentaba poco más de 52 millones de familias, de las cuales 23,9 millones $(45,8 \%)$ eran dirigidas por padres ocupados, de forma asalariada 0 por cuenta propia, dentro de este grupo, al considerar las familias que presentan niños entre 10 y 16 años, el total se reduce a 11,4 millones, siendo que $52,8 \%$ de ellas eran dirigidas por cuenta propia, dentro de este recorte, hay 3,9 millones de niños ocupados en diferentes formas de trabajo, de la cuales $48,8 \%$ tienen hasta 14 años de edad. En cuanto a las características de trabajo, estas se concentran principalmente en las ocupaciones no remuneradas $(45 \%)$, sin registro en cartera $(28,3 \%)$ y en el sector no agrícola-54\% ${ }^{(19)}$.

Al analizar el ingreso familiar de los padres de los estudiantes que trabajan fuera de casa la media salarial fue de $U \$ 338,40^{(10)}$ [2,06 salarios mínimos] siendo que para la mitad de esos sujetos, los ingresos fueron hasta U\$206,57 [1,25 salarios mínimos]. Al comparar con el grupo de niños/adolescentes que no trabajan fuera del hogar, la media del ingreso familiar fue hasta U\$424,74 [2,58 salarios mínimos] y para la mitad de ellos los ingresos fueron hasta U\$ 363,84 [2,21 salarios mínimos]. La prueba $t$ de student mostró diferencia significativa entre los dos grupos, de los que trabajaban fuera de casa y de aquellos que no trabajaban $(p=0.003)$. Fueron comprobadas diferencias de
Caracterización socioeconómica y demográfica de niños y adolescentes que estudian y trabajan simultáneamente fuera de casa Silveira RCP, Ribeiro LC, Secco IAO, Robazzi MLCC 
valores recibidos por los niños/adolescentes por su trabajo fuera de casa $[0,28$ salarios mínimos] y para aquellos que solamente ayudaban en casa [0,05 salarios mínimos] en ambos casos ellos recibieron una cantidad en dinero menor a la que es recibida por un adulto en las mismas condiciones; esto es evidenciado por los valores irrisorios de $U \$ 2,34$ y U\$0,93/mes, mostrando la explotación a que se someten en el trabajo. Posiblemente este hecho ocurra porque ellos no saben, todavía, valorizar su trabajo y aceptan recibir gratificaciones, a pesar de que no deberían trabajar, consecuentemente no recibirían dinero debido a la ilegalidad del trabajo infantil en el Brasil.

La problemática del trabajo y su inserción en la vida de los niños/adolescentes se encuentra presente, particularmente en familias menos favorecidas económicamente. La participación de estas personas en la fuerza de trabajo es una situación social que se viene manteniendo como un problema todavía no resuelto, tanto en países desarrollados como en vías de desarrollo. Los niños comienzan desde muy temprano a auxiliar a sus padres dentro como fuera de casa para contribuir con el ingreso familiar y dedicando poco tiempo o nada para los estudios, volviéndose mano de obra barata no calificada ${ }^{(14)}$.

Las familias brasileñas, consideran normal que los niños/adolescentes trabajen para ayudar con los ingresos familiares; no se sienten infractores al explotar la mano de obra infantil y hasta se apropian de discursos como es mejor pedir que robar, es mejor trabajar que estar en las calles intentando justificar esta opción ${ }^{(10)}$.

El presente estudio mostró motivos para el inicio del trabajo fuera de casa y que son: ayudar a los padres para contribuir con los ingresos familiares (12 casos - 33\%) y ganar dinero (cuatro casos - 11,1\%) entre otros.

En el último informe de la $\mathrm{OIT}^{(5)}$ destacó la importancia de invertir en la educación de las niñas como una forma eficaz de combatir la pobreza. Las niñas que reciben educación tienen mayores probabilidades de recibir salarios más altos en su vida adulta, de casarse más tarde, de tener hijos y que estos sean más saludables, además de ejercer un mayor poder en las decisiones familiares. Además de eso es más probable que las madres con educación garanticen la educación de sus propios hijos, contribuyendo así a evitar el trabajo infantil en el futuro.

En fin, las condiciones de pobreza y exclusión social, consideradas como fuentes generadoras o facilitadoras de la inserción temprana de los niños en el trabajo son apenas contingentes al curso de desarrollo capitalista, en que contradictoriamente se mezclan: la robótica, globalización de los mercados, alta competitividad empresarial y la miseria social creciente. La ocurrencia de esa combinación puede ser detectada en varias cadenas productivas, que en algunos de sus puntos, disfrutan la super explotación del trabajo informal, externalizado, incluyendo la mano de obra infanti|(20).

Así, es importante que la enfermería se atienda esta realidad en que el conocimiento de la salud del trabajador se convierte en el momento actual aún más necesario en la formación de enfermeras para el aumento de la duración de la vida en el trabajo ${ }^{(21)}$, de la super explotación del trabajo informal y de la inserción temprana de los niños en el trabajo.

\section{CONCLUSION}

De los 36 niños/adolescentes que informaron trabajar fuera de casa, la edad media fue de 11,9 años, en actividades que pueden ser consideradas de menor valor social. Recibieron en promedio ingresos de 0,28 salarios mínimos por mes. Con el salario recibido, auxiliaban el incremento del ingreso familiar y además gastar con ellos mismos.

En cuanto los ingresos de los padres/familia se incrementaban, la presencia de los niños/adolescentes trabajando fuera de casa disminuía y viceversa, mostrando una relación próxima entre el trabajo desarrollado y los ingresos familiares insatisfactorios, lo que lleva a los niños/adolescentes a trabajar para ayudar en los gastos de la casa. Así, en el futuro, esos niños/adolescentes podrían tornarse adultos con baja calificación profesional, y que podrían tener dificultades para competir en el mercado de trabajo.

Esta investigación puede contribuir para aumentar el conocimiento del gobierno, educadores, y profesionales en general, referente al problema. Es importante que se atienda esta realidad, aprender a obtener las informaciones adecuadas sobre la situación vivida por los niños/adolescentes trabajadores para que los datos nacionales sean más fidedignos.

La erradicación del trabajo infantil y del adolescente exige respuestas a corto y mediano plazo como la mejoría de las condiciones sociales de los niños y adolescentes que son explotados en el trabajo, además de respuestas a largo plazo, como un proceso de concientización para romper las actuales barreras culturales, en las cuales la sociedad acepta esta condición con naturalidad.

\section{REFERENCIAS}

1. Brasil. Lei n. 8.069, de 13 de julho de 1990. Dispõe sobre o Estatuto da Criança e do Adolescente (ECA) e dá outras providências [Internet]. Brasília; 1990 [citado 2010 fev. 12]. Disponível em: http://www.planalto.gov.br/ccivil_03/leis/L8069. htm
2. Organização Pan-Americana de Saúde (OPAS). Programa de Saúde Materno-Infantil: o marco conceptual da saúde integral ao adolescente e de seu cuidado. Brasília; 1990. 
3. Agencia Brasil. Idade mínima de 16 anos para trabalhar é mantida após rejeição de PEC na Câmara [Internet]. 2009 [citado 2010 jan. 17]. Disponível em: http://agenciabrasil.ebc. com.br/noticia/2009-08-25/idade-minima-de-16-anos-para-

-trabalhar-e-mantida-apos-rejeicao-de-pec-na-camara

4. Organização Internacional do Trabalho (OIT). Pomovendo o trabalho decente. Dia Mundial contra o Trabalho Infantil [Internet]. Genebra; 2011 [citado 2011 nov. 2]. Disponível em: http://www.oit.org.br/content/dia-mundial-contra-o-trabaIho-infantil-2011

5. Organização das Nações Unidas no Brasil (ONU). OIT diz que crise aumenta o risco de que meninas entrem no trabalho infantil [Internet]. Brasília; 2009 [citado 2009 set. 9]. Disponível em: http://www.onu.org.br/oit-diz-que-crise-aumenta-o-risco-de-que-meninas-entrem-no-trabalho-infantil/

6. Organização Internacional do Trabalho (OIT). Trabalho infantil e educação: o desafio do trabalho infantil [Internet]. Genebra; 2008 [citado 2008 jul. 2]. Disponível em: http://www.ilo.org/ public/portugue/region/eurpro/lisbon/pdf/120608_factsheet.pdf

7. Fischer FM, Teixeira LR, Latorre MRDO, Martins IS, Oliveira DC, Costa LAR, et al. Acidentes de trabalho entre estudantes do ensino fundamental e médio das cidades de Santo Antonio do Pinhal e Monteiro Lobato, SP. In: Westphal MF, organizador. Violência e criança. São Paulo: EDUSP; 2002. p. 267-79.

8. Instituto Brasileiro de Geografia e Estatística (IBGE). Síntese de Indicadores Sociais: uma análise das condições de vida da população brasileira [Internet]. Rio de Janeiro; 2010 [citado 2011 nov. 3]. Disponível em: http://www.ibge.gov.br/home/ estatistica/populacao/condicaodevida/indicadoresminimos/ sinteseindicsociais2010/SIS_2010.pdf

9. Brasil. Ministério Público do Trabalho; Procuradoria Regional do Trabalho da 7ạ Região/CE; Assessoria de Comunicação Social. Trabalho infantil no Brasil: Ceará é o quarto no ranking. Diário do Nordeste [Internet]. 2008 abr. 21 [citado 2009 jun. 16]. Disponível em: http://www.prt7.mpt.gov.br/mpt_na_midia/2008/abril/21_04_08_DN_trab_infantil_ceara_quarto. htm

10. International Labor Organization (ILO). A future without child labour: global report

11. [Internet]. Geneva; 2002 [cited 2010 Jan 19]. Available from: http://www.ilo.org/wcmsp5/groups/public/---dgreports/-

--dcomm/---publ/documents/publication/wcms_pu-

bl_9221124169_en.pdf
12. Ribeirão Preto. Secretaria Municipal da Educação. Educação em números: Rede Municipal de Ensino [Internet]. Ribeirão Preto; 2007 [citado 2008 ago. 7]. Disponível em: http:// www.ribeiraopreto.sp.gov.br/seducacao/i15atend05.php

13. Conselho Nacional de Saúde. Resolução n. 196, de 10 de outubro de 1996. Dispõe sobre diretrizes e normas regulamentadoras de pesquisas envolvendo seres humanos. Bioética. 1996;4(2 Supl):15-25.

14. Silveira RCP, Robazzi MLC. As crianças e os adolescentes trabalhadores acidentados no trabalho e atendidos em Unidade Básica Distrital de Saúde em Ribeirão Preto (SP). Ciênc Cuidado Saúde. 2006;5 (2):158-65.

15. Kassouf AL. O que conhecemos sobre o trabalho infantil? Nova Economia. 2007;17 (2):323-50.

16. Facchini LA, Fassa AG, Dall'Agnol M, Maia MFS. Trabalho infantil em Pelotas: perfil ocupacional e contribuição à economia. Ciênc Saúde Coletiva. 2003;8(4):953-61.

17. Asmus CIRF, Raymundo CM, Barker SL, Pepe CCCA, Ruzany $\mathrm{MH}$. Atenção integral à saúde de adolescentes em situação de trabalho: lições aprendidas. Ciênc Saúde Coletiva. 2005;10(4):953-60.

18. Albernaz A, Ferreira FHG, Franco C. Qualidade e equidade no ensino fundamental brasileiro. Pesq Planej Econ. 2002;32(3):453-76.

19. Ferro AR. Jovens e adultos no mercado de trabalho: impacto dos programas de transferência condicional de renda e uma análise agregada usando séries temporais [tese doutorado]. Piracicaba: Escola Superior de Agricultura Luiz de Queiroz, Universidade de São Paulo; 2007.

20. Cacciamali MC, Tatei F. Trabalho infantil e o status ocupacional dos pais. Econ Polit. 2008;28(2):269-90.

21. Ferreira MAF. Trabalho infantil e produção acadêmica nos anos 90: tópicos para reflexão. Estudos Psicol. 2001;6 (2):213-25.

22. Monteiro SM, Santos EV, Kawakami LS, Wada M. O ensino de vigilância à saúde do trabalhador no Curso de Enfermagem. Rev Esc Enferm USP. 2007;41(2):306-10.

\section{Agradecimientos}

A la Coordenação de Aperfeiçoamento de Pessoal de Nivel Superior (CAPES) por el financiamiento de la investigación.

Correspondência: Renata Cristina da P. Silveira Rua Sebastião Gonçalves Coelho, 400 - Chanadour CEP 35501-296 - Divinópolis, MG, Brasil 